\title{
A Lithium Experiment in the Program of Solar Neutrino Research.
}

\author{
A.Kopylov, I.Orekhov, V.Petukhov, A.Solomatin \\ Institute of Nuclear Research of Russian Academy of Sciences
}

\begin{abstract}
The experiments sensitive to pp-neutrinos from the Sun are very perspective for the precise measurement of a mixing angle $\theta_{12}$. A $\nu \mathrm{e}^{-}$scattering experiment (Xmass) and/or a charged-current experiment (the indium detector) can measure the flux of electron pp-neutrinos. One can find the total flux of pp-neutrinos from a luminosity constraint after the contribution of ${ }^{7} \mathrm{Be}$ and $\mathrm{CNO}$ neutrinos to the total luminosity of the Sun are measured. The radiochemical experiment utilizing a lithium target has the high sensitivity to the CNO neutrinos, thus, it has a good promise for the precise measurement of a mixing angle and for the test of a current theory of the evolution of the stars.
\end{abstract}

The main issue addressed by a currently developed lithium detector is the measurement of the contribution of a CNO cycle to the total luminosity of the Sun. According to a Standard Solar Model 1 1 this should be on the level of $1 \%$. The results of gallium experiments 2] give the limits less than $3.5 \%$ (on a $1 \sigma$ level) and $7 \%$ (on a $3 \sigma$ level). Lithium experiment is potentially capable to achieve the level of $0.5 \%(1 \sigma)[3$. Why it is important to reach this level of accuracy? According to a solar model [4] during the evolution of the Sun the concentration of ${ }^{12} \mathrm{C}$ in the central region of the Sun has been diminished as a result of a burning it in a $\mathrm{CNO}$ cycle while the concentration of ${ }^{14} \mathrm{~N}$ has been increased as a product of a CNO cycle, see Fig.1. It is very interesting to test this experimentally. The accurate measurement of the fluxes of the CNO neutrinos will provide the answer to this question. This will be useful also as a general test of a current theory of the evolution of the stars.

Another issue is a more accurate determination of a mixing angle $\theta_{12}$. Of the two parameters of neutrino oscillations $\Delta \mathrm{m}_{12}^{2}$ and $\theta_{12}$ the KamLAND experiment [5] measured the first one using the reactor antineutrinos while the second has been found mainly upon the results of the solar neutrino experiments [6] [11. The important point is that for KamLAND both signs of $\Delta \mathrm{m}_{12}^{2}$ are acceptable while the results of the solar neutrino experiments are compatible only with a positive sign (the mass eigenstate with the larger electron neutrino component has a smaller mass). The determination of the sign of $\Delta \mathrm{m}_{12}^{2}$ would be a great fundamental result because this will be the first step in the identifying a neutrino mass hierarchy. The current interpretation of the results of the solar neutrino experiments is heavily dependent on a very essential point - that our understanding of the neutrino oscillations in matter (MSW effect [12]) is correct in a sense that there are no missing points in the currently accepted description of this process. Although the agreement of all data in the framework of this consideration is very impressive, the general opinion is that still it requires some manifestation in a direct experiment [13. This is why we need more data on 
neutrino oscillations. One of the ways would be to measure with a very high precision a mixing angle for reactor antineutrinos and for solar neutrinos. If there is any even a tiny difference it will denote unambiguously that something is missing in our present understanding of neutrino oscillations in matter. The main reason why Kamland turned out to be rather limited in the determination of a mixing angle is not an optimal distance from a reactor [14.

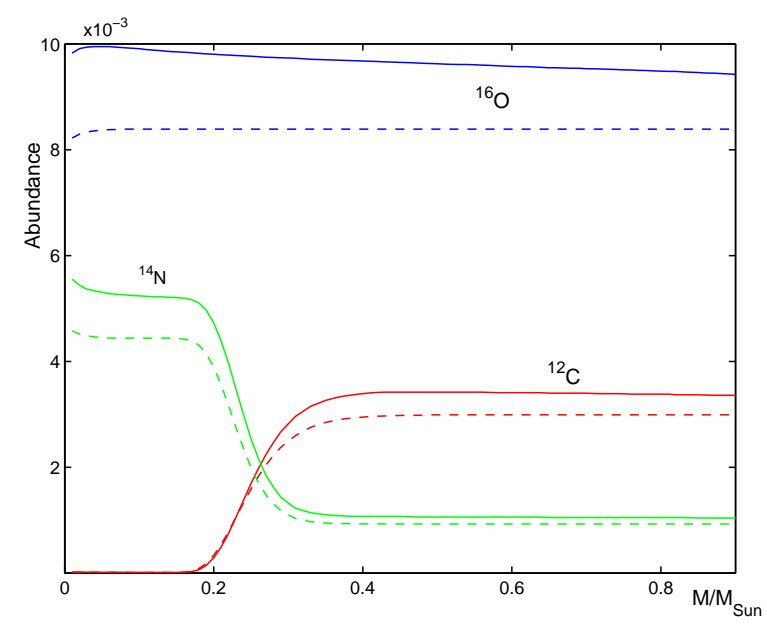

Figure 1: The abundances of ${ }^{12} C$ and ${ }^{14} N$ in the interior of the Sun. The solid lines - with diffusion in the matter of the Sun, the dashed lines - without diffusion.

One can obtain much higher accuracy for a mixing angle if to place the detector in the point of a maximal oscillation effect (minimum in the oscillation curve). The (SADO) detector [15] located in Mt. Komagatake at the distance $54 \mathrm{~km}$ from a reactor complex Kashivazaki-Kariva NPP in Japan can reach a world-record accuracy on $\sin ^{2} \theta_{12} \approx 2 \%(\approx 3 \%)$ at $68.27 \% \mathrm{CL}$ by $60 \mathrm{GW} \cdot \mathrm{kt} \cdot \mathrm{yr}$ (20 GW·kt·yr) operation. The important point also is that this experiment (SADO) would provide a direct prove that the minimum of the oscillation curve really exist. The present data of KamLAND are very compelling to accept this fact, but it would be good to have a direct experimental prove. Is there any possibility to achieve a similar sensitivity in the solar neutrino experiments? Let us look at the results of SNO experiment [1]. From the ratio of the flux of electron neutrinos (found from a charged-current interaction) to the total flux of neutrinos from the Sun (found from a neutral-current interaction) one obtained the value of $\theta_{12}$. The fluxes are determined with the statistical uncertainties of about $4 \%$ and systematic ones of about $5 \%$. The current uncertainties in the determination of $\theta_{12}=33.9_{-2.2}^{+2.4}$ are mainly limited by these numbers.

One can get a further increase of accuracy by means of a substantial improvement in the measurement of the neutrino fluxes. There are several possibilities, which are under development now. Xmass project [16] is trying to utilize the re- 

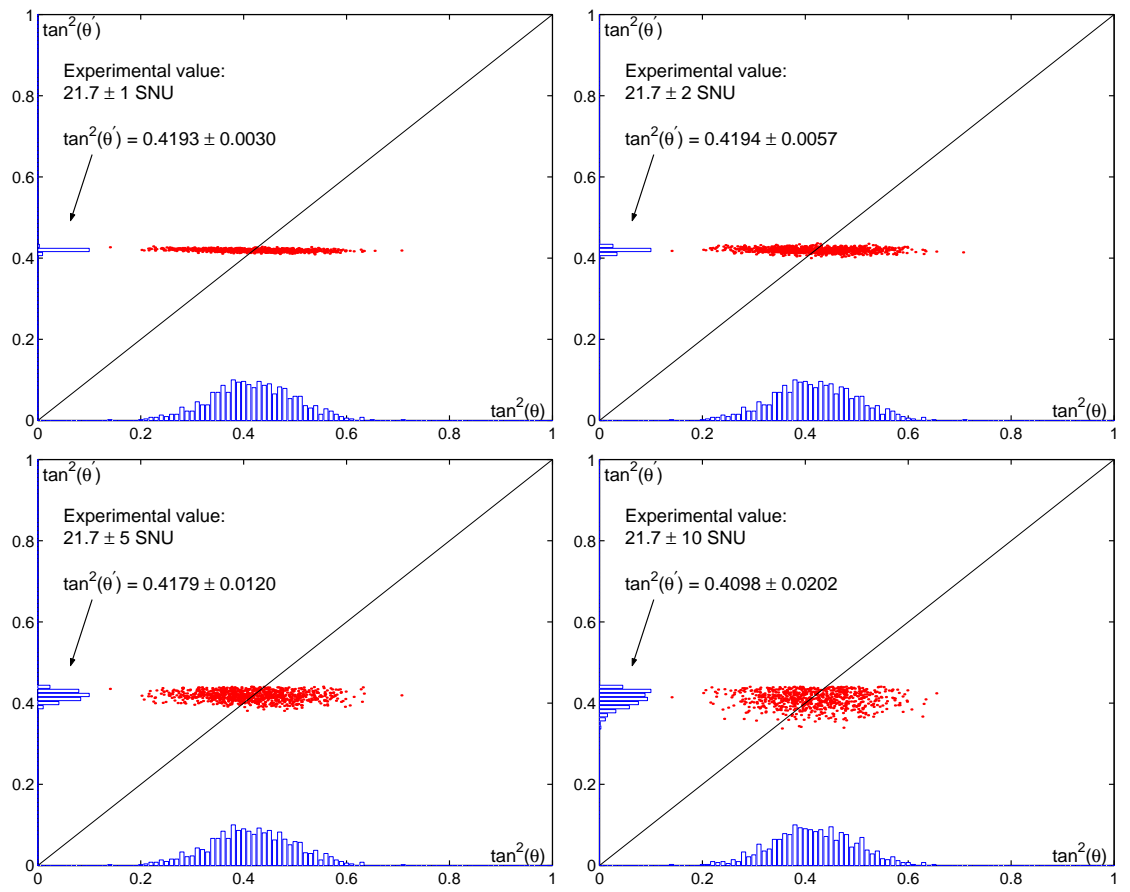

Figure 2: The accuracy of the evaluation of $\theta^{\prime}$ for different uncertainties of a lithium experiment. The distribution on $\mathrm{Y}$-axis is obtained with the Luminosity Constraint applied to the data.

action of $\nu \mathrm{e}^{-}$scattering on Xe. Here one attempts to observe a clear signal from the low energy pp neutrinos. The aim is to reach the accuracy of about $1-2 \%$ for the flux of electron pp neutrinos. Another approach suggested by R.Raghavan [17. is to use a charged-current interaction, which can give a clear signal for the electron pp-neutrinos. Because the cross-section of the neutrino capture reaction by a proposed ${ }^{115}$ In target has large uncertainties, this will need a very powerful neutrino source to calibrate the detector. Both projects have the aim to measure the flux of electron pp neutrinos to improve the accuracy in determination of a mixing angle. Following the strategy realized in SNO, it would be very useful to know the total flux of pp neutrinos also. We suggest a nonstandard approach. Instead of measuring directly the total flux of pp-neutrinos, we propose to determine the flux from a luminosity constraint by evaluating the contribution of non-pp neutrinos $\left({ }^{7} \mathrm{Be}\right.$ and $\left.\mathrm{CNO}\right)$. If to measure the flux of ${ }^{7}$ Be neutrinos with the accuracy of about $10 \%$ and the flux of CNO neutrinos - with the accuracy of about $30 \%$ this will enable to determine the total flux of pp-neutrinos with the accuracy of about $1 \%$ [3]. The low weights of the neutrino generating thermonuclear reactions in the total luminosity of the Sun explain the high tolerant uncertainties for ${ }^{7} \mathrm{Be}$ and $\mathrm{CNO}$ neutrinos. Thus by measuring 
the flux of CNO neutrinos, a lithium experiment will give an essential ingredient - the total flux of pp-neutrinos generated in the Sun, which will enable to improve the accuracy in the determination of a mixing angle. A nontrivial moment in this consideration is the following. Lithium detector is measuring the flux of electron neutrinos coming to the Earth, i.e. with the attenuation factor due to the oscillation effect. This factor is the function of a mixing angle. As the input data we use a mixing angle with the presently obtained uncertainties. After the evaluation of the contribution of CNO cycle to a total luminosity of the Sun, we can find precisely the total flux of pp-neutrinos. (Here we assume that by the time a lithium experiment collects data Borexino and KamLAND will measure the flux of ${ }^{7} \mathrm{Be}$ neutrinos with the accuracy of at least $10 \%$ what will enable to find the contribution of the ${ }^{7} \mathrm{Be}$ neutrino generated reactions to a total luminosity of the Sun with the uncertainty $<1 \%$.) Then by comparing the flux of pp-neutrinos obtained from the data of $\nu \mathrm{e}^{-}$scattering experiment (Xmass) with the total flux of pp-neutrinos we can find precisely a mixing angle as the result on the output. This iterative procedure becomes possible because the weight of the CNO cycle to the total luminosity of the Sun is small (about $1 \%$ ), so that the input uncertainties of a mixing angle (about 10\%) weakly interfere with the output uncertainties (a few percent), see Fig.2. We presented the results of the calculation according to this scheme in [18] where we described the detailed procedure of the calculation.

G.Zatsepin, V.Kuzmin [19] and J.Bahcall 20] were first who proposed a lithium detector. Lithium has a high and precisely calculated cross-section for the neutrino capture reaction because the transition is super allowed. First shown by G.Domogatsky [21] and later calculated more precisely by J.Bahcall 22 is a non-trivial effect, that due to a thermal broadening in the central region of the Sun the ${ }^{7} \mathrm{Be}$ neutrinos contribute significantly to the total yield of ${ }^{7} \mathrm{Be}$ in a lithium target. One can see the production rates for lithium in Table 1.

Table 1: Standard Model Predictions (BP2000): solar neutrino fluxes and neutrino capture rates without neutrino oscillations, with $1 \sigma$ uncertainties from all sources (combined quadratically).

\begin{tabular}{|c|c|c|c|c|}
\hline Source & Flux $\left(10^{10} \mathrm{~cm}^{-2} \mathrm{~s}^{-1}\right)$ & $\mathrm{Cl}(\mathrm{SNU})$ & $\mathrm{Ga}(\mathrm{SNU})$ & $\mathrm{Li}(\mathrm{SNU})$ \\
\hline $\mathrm{pp}$ & $5.95\left(1.00_{-0.01}^{+0.01}\right)$ & 0.0 & 69.7 & 0.0 \\
\hline pep & $1.40 \times 10^{-2}\left(1.00_{-0.015}^{+0.015}\right)$ & 0.22 & 2.8 & 9.2 \\
\hline hep & $9.3 \times 10^{-7}$ & 0.04 & 0.1 & 0.1 \\
\hline${ }^{7} \mathrm{Be}$ & $4.77 \times 10^{-1}\left(1.00_{-0.10}^{+0.10}\right)$ & 1.15 & 34.2 & 9.1 \\
\hline${ }^{8} \mathrm{~B}$ & $5.05 \times 10^{-4}\left(1.00_{-0.16}^{+0.20}\right)$ & 5.76 & 12.1 & 19.7 \\
\hline${ }^{13} \mathrm{~N}$ & $5.48 \times 10^{-2}\left(1.00_{-0.17}^{+0.21}\right)$ & 0.09 & 3.4 & 2.3 \\
\hline${ }^{15} \mathrm{O}$ & $4.80 \times 10^{-2}\left(1.00_{-0.19}^{+0.25}\right)$ & 0.33 & 5.5 & 11.8 \\
\hline${ }^{17} \mathrm{~F}$ & $5.63 \times 10^{-4}\left(1.00_{-0.25}^{+0.25}\right)$ & 0.0 & 0.1 & 0.1 \\
\hline Total & & $7.6_{-1.1}^{+1.3}$ & $128_{-7}^{+9}$ & $52.3_{-6.0}^{+6.5}$ \\
\hline
\end{tabular}

By the total production rate $25 \mathrm{SNU}$ expected in case of neutrino oscillation 
with the presently obtained parameters, $8 \mathrm{SNU}$ is the contribution of neutrinos from a CNO cycle, i.e. approximately $30 \%$ while the contribution of a CNO cycle to the total luminosity of the Sun is only $1.5 \%$. One studied a radiochemical lithium detector as a perspective one since long ago [23]. The main problems were the difficulties in the extraction procedure of ${ }^{7} \mathrm{Be}$ and in the counting of a few atoms of ${ }^{7} \mathrm{Be}$ extracted from the target. The present status of the work on a lithium project is the following. The work on the laboratory installations enabled to choose the optimal layout of the installation: it has a modular structure -20 modules, each module contains $500 \mathrm{~kg}$ of lithium. By now, we completed the development of the technology of extraction of beryllium from lithium on the laboratory installations. We are planning to perform the counting of ${ }^{7} \mathrm{Be}$ by means of the array of HPGe detectors. Now the work is going on the construction of the first module of the full-scale lithium detector as a pilot lithium installation. The aim is to demonstrate on one module the effective functioning of the technique.

This work was supported in part by a Russian Foundation for Basic Research (project no. 04-02-16678), by the grant of Russia Leading Scientific Schools LSS-1786.2003.2 and by the Program of fundamental research of Presidium of Russian Academy of Sciences "Neutrino Physics". The authors deeply appreciate the very stimulating discussions with G.Zatsepin, L.Bezrukov and V.Kuzmin and express a gratitude to B.Zhuikov for the help in the irradiation of an aluminum sample in a proton beam of a Moscow meson facility which enabled us to complete the work on the development of the technology of the beryllium extraction from a lithium target.

\section{References}

[1] J. N. Bahcall, astro-ph/0402114.

[2] C.Cattadori, Results from Radiochemical Solar Neutrino Experiments XXI International Conference on Neutrino Physics and Astrophysics (Neutrino 2004, Paris, June 14-19, 2004).

[3] A. V. Kopylov et al., Physics of Atomic Nuclei, 67(3), 1182 (2004).

[4] J. N. Bahcall, Neutrino Astrophysics (Cambridge Univ.Press, Cambridge, 1998).

[5] K. Eguchi et al., Phys.Rev.Lett. 90021802 (2003).

[6] B. T. Cleveland et al., Astrophys.J. 496505 (1998).

[7] J. N. Abdurashitov et al., (SAGE Collab.) Pis'ma Zh. Éksp. Teor. Fiz. 95, 181 (2002).

[8] W. Hampel et al., (GALLEX Collab.), Phys.Lett.B 447127 (1999).

[9] E. Belotti et al., (GNO collab.), Nucl.Phys.B (Proc. Suppl.) 9144 (2001). 
[10] Y.Fukuda et al., Phys. Rev. Lett. 865651 (2001).

[11] B.Aharmim et al., (SNO Collab.) nucl-ex/0502021

[12] S. P. Mikheyev and A. Yu. Smirnov, Sov.J.Nucl.Phys. 42913 (1985); L. Wolfenstein, Phys.Rev. D17 2369 (1978).

[13] I am grateful to Carlos Peña-Garay for the illuminating discussion on this point.

[14] S. Choubey, hep-ph/0402288

[15] H. Minakata et al., hep-ph/0407326

[16] M. Nakahata, Report at International Conference (NOON-2004, Japan).

[17] R. Raghavan, Report at International Conference (TAUP-2005, Spain).

[18] A. Kopylov and V. Petukhov, hep-ph/0308004

[19] G. T. Zatsepin and V. Kuzmin, in Proceedings of the $9^{\text {th }}$ ICRC, 1024 (London, 1965).

[20] J. N. Bahcall, Phys.Lett., 3332 (1964).

[21] G. V. Domogatsky, Preprint No. 153, FIAN (1969) Moscow.

[22] J. N. Bahcall, Rev.Mod.Phys. 50881 (1978).

[23] J. K. Rowly, Proc.Conf.on Status and Future of Solar Neutrino Research, BNL, Jan. 5-7, 265 (1978)

E. Veretenkin, V. Gavrin, E. Yanovich, Russian Journal "Atomic Energy" 88 (N1) 65 (1985)

A. V. Kopylov, A. N. Likhovid, E. A. Yanovich, G. T. Zatsepin, Proc. Intern. School "Particles and Cosmology", Baksan Valley, Russia, 2227 April 1993. Editors: E. N. Alekseev, V. A. Matveev, Kh. S. Nirov, V. A. Rubakov, (World Scientific, Singapore-New Jersey-London-Hong Kong) 63 (1993)

S. Danshin, G. Zatsepin, A. Kopylov et al., Part.Nucl. 285 (1997)

M. Galeazzi, G. Gallinaro, F. Gatti et al., Phys.LettersB 398187 (1997)

A. V. Kopylov, in Proc.Intern.Conf. on Nonaccelerator New Physics, Dubna, Russia, June 28 - July 3, 1999, (Russian Journal "Nuclear Physics"), 63 (N7) 1345 (2000) 